\title{
Combination of Structured Illumination Microscopy with Hyperspectral Imaging for Cell Analysis
}

\author{
Guoxuan Liu ${ }^{1, \ddagger}$, Huaidong Yang1, ${ }^{1, *}$, Hansen Zhao², Yinxin Zhang3,*, Sichun \\ Zhang ${ }^{2}$, Xinrong Zhang,*, Guofan Jin ${ }^{1}$ \\ ${ }^{1}$ Department of Precision Instrument, State Key Laboratory of Precision Measurement \\ Technology and Instruments, Tsinghua University, Beijing 100084, China \\ ${ }^{2}$ Department of Chemistry, Beijing Key Laboratory for Microanalytical Methods and \\ Instrumentation, Tsinghua University, Beijing 100084, China \\ ${ }^{3}$ Key Laboratory of Opto-electronic Information Technology, Ministry of Education, TianJin \\ University, Tianjin, 300072, China \\ $\ddagger$ These authors contributed equally. \\ *Huaidong Yang: yanghd@tsinghua.edu.cn \\ *Yinxin Zhang: yinxin@tju.edu.cn \\ *Xinrong Zhang: xrzhang@tsinghua.edu.cn
}

\section{Supplementary Materials}

\begin{tabular}{|l|l|}
\hline Supplementary Note 1 & Optical configuration of the hyperspectral SIM \\
\hline Supplementary Note $\mathbf{2}$ & Cell culture and fluorescent staining \\
\hline Supplementary Note $\mathbf{3}$ & Capability of hyperspectral SIM \\
\hline Supplementary Note 4 & Data processing \\
\hline Supplementary Figure S1 & $\begin{array}{l}\text { Engineering drawing demonstrating the parameters of the } \\
\text { customized lenslet array }\end{array}$ \\
\hline Supplementary Figure S2 & Determination of the light efficiency for hyperspectral SIM \\
\hline Supplementary Figure S3 & $\begin{array}{l}\text { The hyperspectral SIM raw image and the restored datacube of } \\
\text { the SIM raw image }\end{array}$ \\
\hline Supplementary Figure S4 & $\begin{array}{l}\text { Determination of the spectral resolution of the hyperspectral } \\
\text { system }\end{array}$ \\
\hline Visualization 1 & $\begin{array}{l}\text { Time-lapse image sequence of 590 nm wavelength } \\
\text { (mitochondria) in the hyperspectral SIM imaging result for the } \\
\text { two-channel HEPG2 cell }\end{array}$ \\
\hline Visualization 2 & $\begin{array}{l}\text { Datacube of the first time point in the time-lapse hyperspectral } \\
\text { SIM imaging result of the two-channel HEPG2 cell }\end{array}$ \\
\hline Visualization 3 & $\begin{array}{l}\text { Datacube of the hyperspectral SIM imaging result of the } \\
\text { spectral-overlapped four-channel HEPG2 cell }\end{array}$ \\
\hline
\end{tabular}




\section{Supplementary Note}

\section{Optical Configuration of the Hyperspectral SIM}

The schematic diagram of the hyperspectral SIM setup is illustrated in Figure 1a of the manuscript, consisting of a digital micromirror device (DMD)-based structured illumination system, a commercial microscope body and an integral field spectroscopy (IFS) system.

In the DMD-based structured illumination system, laser beams of different wavelengths are employed as the light source. After being collimated and expanded, the laser beams are coupled with a dichroic mirror and then used as the incident beams of the digital micromirror device (DMD) (W4100Kit, Wintech). The micromirrors periodically arranged on the DMD planes diffract the incident beams into multiple diffraction orders. By adjusting the incident angles of different wavelengths, we can obtain an emergence diffraction order for each wavelength that is perpendicular to the DMD plane and near the blaze direction. This diffraction order is selected for the subsequent illumination and further split into multipleorder spatial spectra by the periodic modulation patterns loaded on the DMD. After passing through a spatial filter system, a spatial filter with six hexagonally arranged holes ${ }^{1}$ filters out all the spatial spectrum orders except for the \pm 1 order in three different illumination directions. The residual \pm 1 order spatial spectrum are then focused on the back-focal plane of the objective lens, forming two collimated beams that interference with each other at the sample plane and generating a periodic pattern for structured illumination of the sample. A polarizer inserted at a proper angle is used to maintain the near-equal contrast of the illumination patterns in different directions ${ }^{2}$. Owing to the wavelength-independent characteristic of DMD, this DMD-based illumination system enables simultaneous structured illumination of the sample using multiple wavelengths.

The sample stimulated with the multiwavelength structured illumination pattern is collected by a commercial microscope body (Ti-E, Nikon), forming a multicolor sample image at the tube lens focal plane (i.e., the image plane of the microscope). This multicolor sample image is then integrated into the following IFS subsystem.

In the IFS subsystem, an additional microscope system is included that consists of an objective lens (CFI TU Plan Fluor 5×, Nikon) and a tube lens (TTL200-A, Thorlabs). This system first premagnifies the multicolor sample image. Then, in the image plane of the additional microscope system, a lenslet array is mounted that performs discrete sampling of the premagnified image. Energy passing through each lenslet corresponds to the intensity of a pixel in the discrete sample image (Figure 1b). This premagnification process guarantees discrete sampling that meets the Nyquist criterion, and the resolution of the discrete sample image reaches the diffraction limit. Next, each lenslet focuses on the corresponding pixel of the discrete sample image, transforming the densely packed discrete sample image into a sparse point array at the focal plane of the lenslet array (Figure 1c). A spectroscope system consists of a collimator (EF $50 \mathrm{~mm}$ f/1.8 STM, Canon), a prism (SM1W1122, Thorlabs) and a collector (AF Zoom-NIKKOR 70-300 mm f/4-5.6 G, Nikon, the focal length was adjusted to about $100 \mathrm{~mm}$ in the experiment) further disperses the sparse point array into an individual spectral line array (Figure 1d), in which each spectral line corresponds to the spectrum of a pixel in the discrete sample image and the lines do not overlap. Thus, the IFS subsystem maps the three-dimensional datacube $(x, y$, and $\lambda)$ of the multicolor sample image into the detector plane. After acquiring the spectral line array with a scientific CMOS camera (sCMOS, Zyla 4.2 Plus, Andor) placed in the focal plane, a hyperspectral SIM raw image is obtained. The datacube of the SIM raw image can be further restored from the spectral line array, substantially achieving snapshot spectral imaging of a SIM raw image.

In practical experiments, $488 \mathrm{~nm}$ (Sapphire $488 \mathrm{LP}$, Coherent) and $561 \mathrm{~nm}$ (MGL-FN-561, Cnilaser) lasers were used for sample illumination. The fluorescence filter sets used in the microscope are a dichroic mirror (ZT488/561rpc-UF2, Chroma) and an emission filter 
(ZET488/561m, Chroma), which filter out the illumination light while keeping the majority of fluorescence light. In addition, a long-pass filter (FEL0500, Thorlabs) and a short-pass filter (FES0650, Thorlabs) were inserted into the IFS subsystem to determine the detected wavelength range of the hyperspectral SIM setup. In the experiment involving imaging of the BPAEC slide, a 60×/0.5-1.25NA objective lens (CFI Plan Fluor 60XS Oil, Nikon) was used for microscopic imaging; the camera exposure time for capturing a single hyperspectral SIM raw image (the individual line array) was 2 s. In two-channel living Hepg2 cell imaging, however, the camera exposure time was set to $0.3 \mathrm{~s}$ to achieve better dynamic performance. In the four-channel living Hepg2 cell imaging, the detector exposure time was also set to $0.3 \mathrm{~s}$ but a $20 \times / 0.45$ NA objective lens (CFI TU Plan Fluor 20×, Nikon) was applied to achieve a larger focal depth and a larger FOV, guaranteeing that the in-focus signals of the four channels exist in the detected result simultaneously. A customized lenslet array made of fused silica was used in the experiments (Figure S1). The size of the lenslet array was $13 \mathrm{~mm} \times 13$ $\mathrm{mm} \times 1 \mathrm{~mm}$. The subapertures of the lenslets were $40 \mu \mathrm{m} \times 40 \mu \mathrm{m}$ squares. Each lenslet consisted of a planoconvex lens with a $0.1 \mathrm{~mm}$ curvature radius of the convex plane. Different lenslets were arranged orthogonally, as illustrated in Figure S1. The transmittance of the filters and the lenslet array determined that, in the experiment, $61 \%$ percent of the fluorescent light in the FOV of hyperspectral SIM could be eventually detected, which was calculated by comparing the energy in the ROI of a sample image detected by hyperspectral SIM and that of the same sample image detected through another imaging port of the microscope (Figure S2).

\section{Cell Culture and Fluorescent Staining}

HEPG2 cells were cultured in DMEM mixed with 10\% fetal bovine serum (FBS, Gibco) and $1 \%$ penicillin/streptomycin by volume. Cell staining was conducted when the cell coverage reached approximately 30\%. To prepare the stain, the cell culture medium was removed and washed 2 times with Dulbecco's phosphate-buffered saline (DPBS, Gibco). The commercially available fluorescent stain reagents SYTO14 (S7576, Invitrogen), TublinTracker Orange Green 488 (T34075, Invitrogen), WGA Alexa Fluor 594 (A12381, Invitrogen) and MitoTracker Orange CMTMRos (M7510, Invitrogen) were purchased from Thermo Fisher and prepared as stock solutions following the protocols provided. Staining was conducted in Hank's balanced salt solution (HBSS). The optimized final working concentrations for SYTO14, TublinTracker Orange Green, WGA Alexa Fluor 594 and MitoTracker Orange were $500 \mathrm{nM}, 0.83 \mu \mathrm{g} / \mathrm{mL}(2.5 \times), 1 \mu \mathrm{g} / \mathrm{mL}$ and $50 \mathrm{nM}$, respectively. The HBSS and the stock solutions were premixed before being added to the cell culture dish. The cells were incubated with the stain reagents for 1 hour and washed with HBSS 2 times before imaging.

\section{Capability of Hyperspectral SIM}

\section{Spatial Capability}

The spatial resolution ability of hyperspectral SIM is similar to that of conventional SIM. For example, during hyperspectral SIM imaging of BPAEC slides, we used a $60 \times / 1.25 \mathrm{NA}$ objective lens for sample collection. For the central wavelength of the detected wavelength range, which is $575 \mathrm{~nm}$, the corresponding diffraction limit is:

$$
R_{\text {widefeild }}=0.61 \cdot \lambda / N A \approx 281 \mathrm{~nm},
$$

where $R_{\text {widefield }}$ is the spatial resolution of widefield imaging (i.e., the diffraction limit), $\lambda$ equals $575 \mathrm{~nm}$, and NA is the numerical aperture of the objective lens, which equals 1.25. The period of the patterns loaded on DMD was approximately 10 pixels, while the pixel size of the DMD was $10.8 \mu \mathrm{m}$. After spatial filtering and focusing to the back focal plane of the objective lens using a $400 \mathrm{~mm}$ lens, the illumination light formed the required periodic 
illumination patterns with a period of nearly $450 \mathrm{~nm}$. Thus, the theoretical spatial resolution of hyperspectral SIM is:

$$
R_{\text {spat }}=1 /\left(1 / R_{\text {widefield }}+1 / p\right) \approx 173 \mathrm{~nm},
$$

where $R_{\text {spat }}$ is the spatial resolution of hyperspectral SIM and $p$ is the period of the illumination pattern in the sample plane ( $450 \mathrm{~nm}$ as calculated above).

Two important preconditions should be satisfied in practical experiments to approach the theoretical spectral resolution capability. First, the optical design of the prototype should be optimized, especially the IFS system. Second, the discrete sampling of the microscopic image using the apertures of the lenslet array in the IFS system should meet the Nyquist criterion. The former precondition guarantees that the spatial resolution of the image will not degrade during the transmission process. This is an important task that should be completed during further improvements of the prototype. The second precondition guarantees that the spatial resolution of the discrete sampling result reaches the diffraction limit; this precondition was already satisfied in the current prototype. Considering the $60 \times$ magnification of the objective lens, the $1.5 \times$ medium magnification of the microscope body and the $5 \times$ magnification of the extra microscope subsystem in the IFS, the diffraction limit $R_{\text {widefield }}$ in the sample plane will be magnified to $126 \mu \mathrm{m}$ while being discretely sampled by the lenslet array. Thus, the $40 \mu \mathrm{m}$ pitch size of the lenslet array we used meets the Nyquist criterion well in this situation.

Although hyperspectral SIM retains the spatial resolution capability of conventional SIM, the field-of-view (FOV) in hyperspectral SIM is quite different from that determined directly by the detector area in conventional SIM. In hyperspectral SIM, the sample image is transformed into a sparse spectral line array. A considerable part of the detector data throughput is transformed into spectral detection, and blank pixels which we refer to as data redundancy are also reserved for separating the adjacent spectral curves. Both aspects will result in obvious degradation in the FOV. The FOV of hyperspectral SIM can be estimated as follows.

Considering the $50 \mathrm{~mm}$ and $100 \mathrm{~mm}$ focal lengths of the collimator and condenser in the IFS system, the magnification of the spectroscope system, $M_{\text {spec }}$, equals 2 . Thus, the size of the detectable discrete sampled image $l_{\text {img }}$ will be

$$
l_{\text {img }}=N \times p_{d} /\left(p_{L A} \times M_{\text {spec }}\right)=174 \text { pixels, }
$$

where $N$ is the number of pixels in the detector in each dimension, $N$ equals 2048, $p_{d}$ is the pixel size of the detector, and $p_{d}$ equals $6.5 \mu \mathrm{m}, p_{L A}$ is the pitch size of the lenslet array, $p_{L A}$ equals $40 \mu \mathrm{m}, M_{\text {spec }}$ equals 2 as mentioned above. When using the $60 \times$ objective lens for sample collection, the corresponding FOV will be

$$
l_{\text {FOV }}=p_{L A} / M_{o b j} / M_{i} / M_{e} \times l_{i m g}=15.5 \mu m,
$$

where $p_{L A}$ equals $40 \mu \mathrm{m}, M_{o b j}$ is the $60 \times$ magnification of the objective lens, $M_{i}$ is the $1.5 \times$ intermedium magnification of the microscope body, and $M_{e}$ is the $5 \times$ magnification of the additional microscope system in the IFS. Thus, hyperspectral SIM achieves a $15.5 \mu \mathrm{m} \times 15.5$ $\mu \mathrm{m} \times 15.5 \mu \mathrm{m}$ FOV while using a $60 \times$ objective lens. This approach essentially sacrifices the FOV of hyperspectral SIM for spectral detection capability.

For further enhancement of the hyperspectral SIM FOV, efforts can be made to optimize the optical design of hyperspectral SIM. With optimized optical design, we can obtain much narrower spectral line in the hyperspectral SIM raw data and a lower spectroscope magnification $M_{\text {spec }}$ is required, which means we can reduce the blank pixels reserved for separating adjacent spectral lines, minimizing the data redundancy, and taking full advantage 
of the detector data cube. Besides, applying an advanced detector (iKon-XL 231, Andor) with about 4-fold larger data throughput is also a good option for FOV enhancement.

\section{Spectral Capability}

The spectral capability of hyperspectral SIM depends on the filter sets and the dispersion subsystem in IFS. The long-pass and short-pass filters determine the detectable wavelength range (500 $\mathrm{nm}$ to $650 \mathrm{~nm}$ ) of the system. The dispersion subsystem, however, determines the spectral resolution of hyperspectral SIM.

The dispersion subsystem is essentially a spectroscope consisting of a collimator $(f=50 \mathrm{~mm})$, a prism, and a condenser $(f \approx 100 \mathrm{~mm})$ (Figure $1 \mathrm{a})$. The prism is made of NBK7 glass, and the wedge angle is $11^{\circ} 22$ '. According to these parameters, the refracted angle of the prism can be calculated easily using Snell's law. Thus, the length of the spectral line in the detector plane will be

$$
l_{\text {spec }} \approx f_{\text {cond }} \cdot \Delta \alpha=150 \mu \mathrm{m},
$$

where $l_{\text {spec }}$ is the length of the spectral line in the detector plane, $f_{\text {cond }}$ is the focal length of the condenser, and $\Delta \alpha$ is the angle between the chief rays of the $500 \mathrm{~nm}$ and $650 \mathrm{~nm}$ refracted beams of the prism. If the spectral line array is well focused, the full width at half maxima (FWHM) of the PSF in the detector plane will be smaller than the pixel size of the detector $(6.5 \mu \mathrm{m})$. Under this condition, the theoretical spectral resolution of hyperspectral SIM will depend on the sampling rate of the spectral lines, which is:

$$
R_{\text {spec }}=(650 \mathrm{~nm}-500 \mathrm{~nm}) / \frac{l_{\text {spec }}}{2 p_{d}}=13 \mathrm{~nm},
$$

where $R_{\text {spec }}$ is the theoretical spectral resolution of hyperspectral SIM, and $p_{d}$ is the pixel size of the detector.

\section{Temporal Capability}

Hyperspectral SIM achieves snapshot imaging of the SIM raw images and allows the spectral dimension of SIM to be explored without requiring extra exposures. Thus, hyperspectral SIM shares the same temporal capability as conventional single-color SIM. Theoretically, the highest speed of hyperspectral SIM will be one nth of the detector speed limit, where n, which typically equals 9 , is the number of raw images required to reconstruct a super-resolution SIM image. In practical applications, however, to guarantee the SNR of the detected data, the detector usually did not reach its highest working speed. Even so, hyperspectral SIM is still $m$ times faster than conventional SIM using the wavelength-scanning strategy, where $m$ is the total number of wavelength bands to be captured using the wavelength-scanning strategy.

\section{Data Processing}

\section{Calibration of the IFS System}

To extract the datacube of the SIM raw image from the captured spectral line array, we must first calibrate the IFS system, which characterizes the pixel-to-wavelength relation of each spectral line in the detector plane. Here, we employed a wavelength-template-based parallel calibration method. The wavelength template is the hyperspectral SIM raw image (spectral line array) of a homogenous reflective sample (a mirror) illuminated evenly with a monochromatic beam. Owing to the monochromaticity, the spectral line array in a wavelength template is essentially a point array because each spectral line contains only a single pulse at the location corresponding to the illumination wavelength. This point array inherently records the pixel-to-wavelength relation of a certain wavelength of the IFS system. It is further utilized to extract the SIM raw image from the hyperspectral SIM raw image (introduced in 
the subsequent section). Before that, the captured wavelength template should be preprocessed. We preserve the important pixels (pixels in the $5 \times 5$ region centered around the highest intensity pixel of a point) of the wavelength template, which gathers most of the energy of each point while setting the other pixels to zero. Then, the total energy of each point is normalized to eliminate intensity heterogeneity of the reflective sample image while capturing the wavelength template. In this way, we obtain a preprocessed wavelength template. Obtaining the templates of the required wavelengths and the preprocessing are the only necessary steps to achieve calibration.

In the practical calibration process, a super continuous source (SuperK EXTREME EXW12, NKT) was used for widefield illumination of the sample, providing a variable central wavelength and a near-10-nm bandwidth, which can be considered monochromatic in an IFS system with a spectral resolution of $15 \mathrm{~nm}$ (Figure S4). The central wavelength was swept from $500 \mathrm{~nm}$ to $650 \mathrm{~nm}$ at a step size of $5 \mathrm{~nm}$. Thirty-one different wavelength templates were captured. In addition, during calibration, the dichromatic splitter was replaced by a 50:50 beam splitter (BSW10R, Thorlabs), and all the other filters in the microscope and IFS system were removed to prevent the sharp edges of the transmission curves of the filters from affecting the accuracy of the calibration results.

\section{Extracting the Datacube of SIM Raw Images}

After calibration, the datacube of the structured illuminated sample images (SIM raw images) can be extracted from the hyperspectral raw image. Multiplexing the preprocessed wavelength template and a hyperspectral SIM raw image of the sample pixel-by-pixel, the hyperspectral SIM raw image (spectral line array) will be filtered into a sparse point array. In this point array, the total intensity of a point corresponds to the intensity of a pixel in the monochromatic SIM raw image. In this way, we can extract the monochromatic SIM raw images for all wavelengths of interest and construct the required datacube of SIM raw images for the following reconstruction (Figure S3). Occasionally, dust or any of several manufacturing defects may exist in the lenslet array. This can introduce some obvious singlepixel errors in the fixed locations of the imaging result. To eliminate such errors, we recommend $3 \times 3$ mean filtering, especially in certain pixels of the extracted image.

Another thing that should be mentioned is that we sometimes found moiré-fringe-like artifacts in the extracted SIM raw images in the practical experiments, especially when the contrast of the extracted image was low. Such artifacts appear because of inaccurate preprocessing of the wavelength template that selects the important pixels for the point array-which is essentially another sampling process of the captured data. In the future, a more accurate method is required to utilize the inherent pixel-to-wavelength relation of the wavelength template for datacube extraction.

\section{Reconstruction of the Hyperspectral SIM}

After obtaining the datacube of the SIM raw images, we can reconstruct the super-resolution SIM image of each wavelength using the corresponding slices of these datacubes and further rearrange them into a datacube of the super-resolution SIM image. In this operation, the reconstruction process for each wavelength is the same as that used in conventional singlecolor $\mathrm{SIM}^{3}$.

\section{Spectral Unmixing}

Spectral unmixing is a common spectral analysis method and is the key to extracting the single-channel super-resolution images from the spectrum-overlapped datacube. In this work, we applied the linear mixing model (LMM) during spectral unmixing ${ }^{4}$. The LMM ignores the interactions between different fluorescence labels and considers the emission spectral curve of the sample result as a linear combination of the individual spectral curves of all the labels. For each pixel of the super-resolution SIM image, the emission spectral curve was 


$$
x=\sum_{i=1}^{M} \alpha_{i} s_{i}+w,
$$

where $\mathrm{x}$ denotes the total spectrum vector of the pixel, $M$ is the total number of fluorescence labels, $s_{i}$ is the spectrum vector of each individual fluorescence label, $\alpha_{i}$ is the nonnegative abundance of each fluorescence label, and $\mathrm{w}$ is the sum vector of additional detector noise and the background signal of the sample. Before spectral unmixing, the individual spectrum vector $s_{i}$ of the fluorescence labels and the sum vector $\mathrm{w}$ were predetected; consequently, the abundance $\alpha_{i}$ is the only unknown value in the equation. Considering that the dimension of the total spectrum vector $x$ is generally much higher than the total number of fluorescence labels, the equation is an overdetermined equation. The least squares method was applied to calculate the abundance of each fluorescence label. Fluorescence label abundances were calculated for each pixel in the super-resolution SIM image. For each fluorescence label, the abundance distribution consists of its own single-channel super-resolution SIM image. In our practical experiment, the detected wavelengths ranged from $500 \mathrm{~nm}$ to $650 \mathrm{~nm}$, and the spectral resolution was $15 \mathrm{~nm}$. Thus, the effective dimension of the spectrum vectors was 11, which means that at most 11 different fluorescence labels with linearly independent spectra could theoretically be unmixed from the spectral-overlapped datacube.

In the future, for fluorescence labels with heavier spectral overlap, the interactions between different fluorescence labels could no longer be ignored. A suitable nonlinear

unmixing model should be applied in such situations to obtain more accurate unmixing results.

\section{References}

(1) Foerster, R.; Lu-Walther, H.-W.; Jost, A.; Kielhorn, M.; Wicker, K.; Heintzmann, R. Optics Express 2014, 22, 20663-20677.

(2) O’Holleran, K.; Shaw, M. Optics Letters 2012, 37, 4603-4605.

(3) Lal, A.; Shan, C.; Xi, P. IEEE Journal of Selected Topics in Quantum Electronics 2016, 22, 50-63.

(4) Keshava, N.; Mustard, J. F. IEEE Signal Processing Magazine 2002, 19, 44-57. 


\section{Supplementary Figures}
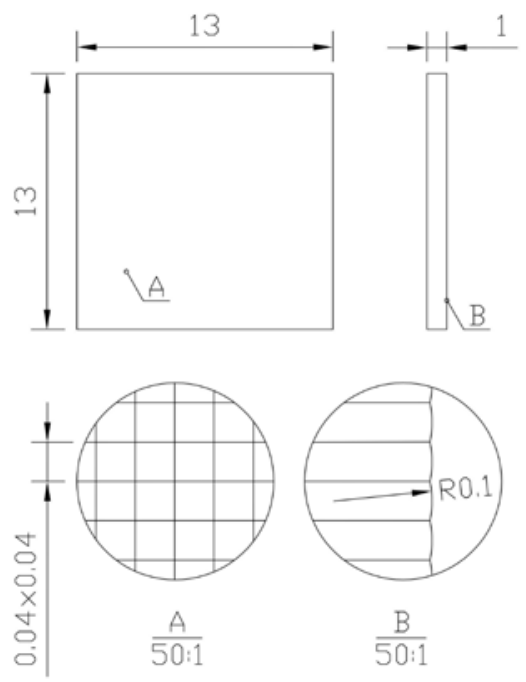

Figure. S1. Engineering drawing demonstrating the parameters of the customized lenslet array. The unit of dimension in the drawing is millimeters.
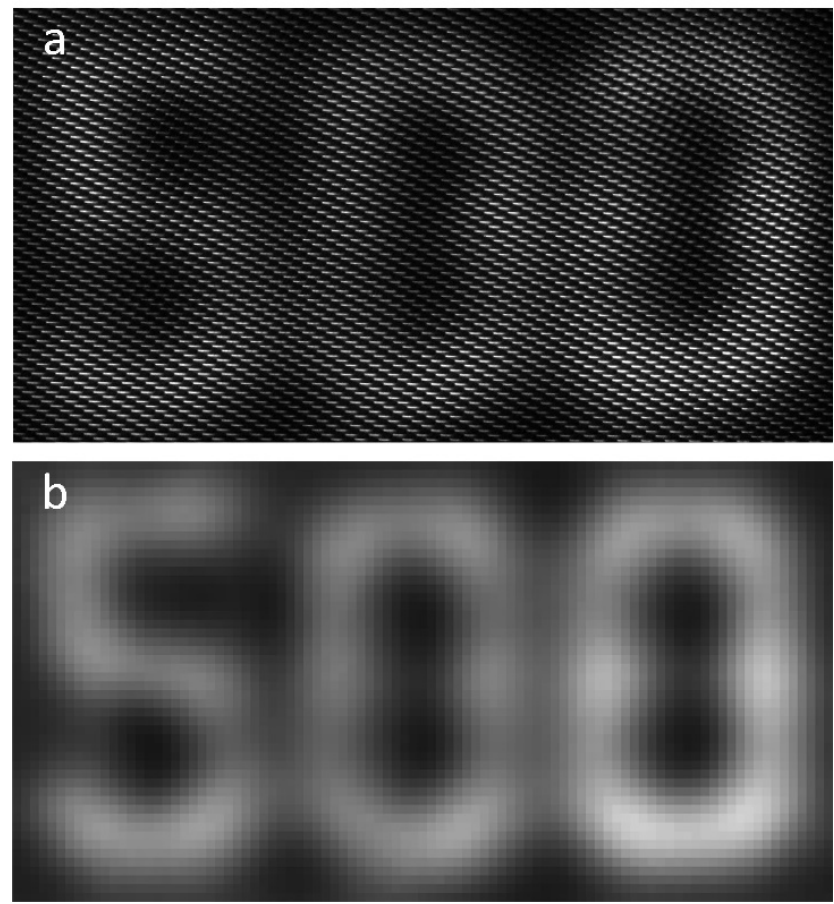

Figure. S2. Determination of the light efficiency for hyperspectral SIM. (a) ROI of a sample image detected through hyperspectral SIM setup. (b) ROI of the same sample image as that in (a), detected through another imaging port of microscope. By comparing the total energy of (a) and (b) after background subtraction, the light efficiency for hyperspectral SIM is determined, which describes the fraction of fluorescence energy in the FOV that eventually reaches the detector. 

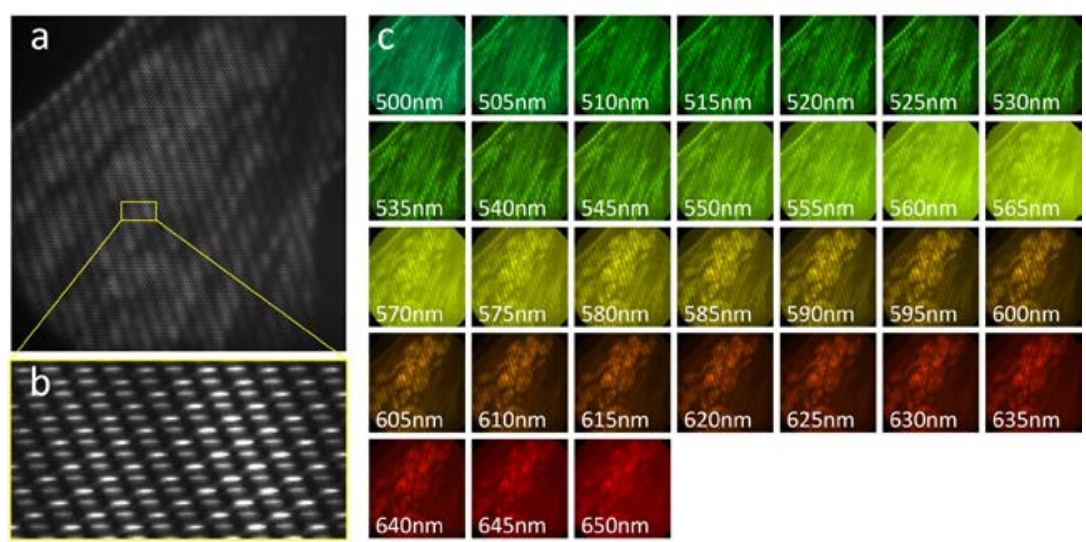

Figure. S3. The hyperspectral SIM raw image and the restored datacube of the SIM raw image: (a) the hyperspectral SIM raw image, i.e., the individual spectral line array; (b) enlarged ROI of the spectral line array; (c) restored SIM raw images of different wavelengths from the hyperspectral SIM raw image. The contrast of these images was adjusted for better illustration.
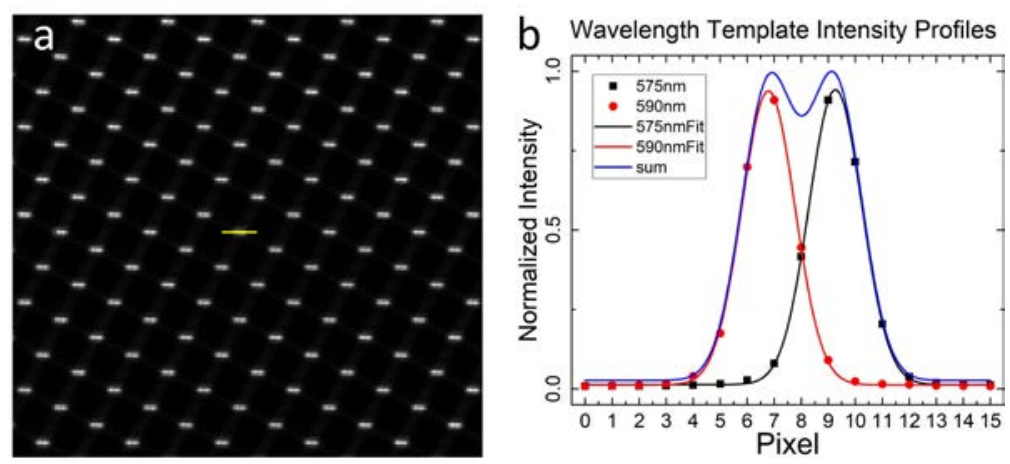

Figure. S4. Determination of the spectral resolution of the hyperspectral system: (a) central region of the superposed wavelength template of $575 \mathrm{~nm}$ and $590 \mathrm{~nm}$; (b) the intensity profile on the selected yellow line. The superposed fitting curve of these two wavelength templates just met the Rayleigh criterion. The adjacent points from two separate wavelengths were just barely resolvable. Thus, their wavelength interval, $15 \mathrm{~nm}$, was considered the spectral resolution of the hyperspectral SIM system. 


\section{Visualizations}

Visualization 1. Time-lapse image sequence of $590 \mathrm{~nm}$ wavelength (mitochondria) in the hyperspectral SIM imaging result for the two-channel HEPG2 cell.

Visualization 2. Datacube of the first time point in the time-lapse hyperspectral SIM imaging result of the two-channel HEPG2 cell. The SIM image of each wavelength in the datacube was normalized for better illustration.

Visualization 3. Datacube of the hyperspectral SIM imaging result of the spectral-overlapped four-channel HEPG2 cell. The SIM image of each wavelength in the datacube was normalized for better illustration. 